\title{
Carga y presión tributaria. Un estudio del efecto en la liquidez, rentabilidad e inversión de los contribuyentes en Ecuador
}

\author{
Tax load and pressure. A study of the effect on the liquidity, \\ profitability and investment of taxpayers in Ecuador
}

Dra. Gabith Miriam Quispe-Fernández es profesora e investigadora de la Universidad Nacional de Chimborazo (Ecuador) (gquispe@unach.edu.ec) (https://orcid.org/0000-0002-7485-3669)

Dr. Dante Ayaviri-Nina es profesor e investigador de la Universidad Nacional de Chimborazo (Ecuador) (dayaviri@unach.edu.ec) (https://orcid.org/0000-0002-3078-1771)

\begin{abstract}
Resumen
Las altas tasas y diferentes impuestos determinan la recaudación tributaria en los diferentes ciclos económicos de las economías; en ese contexto, la investigación analiza el efecto de la carga y presión tributaria en la liquidez, rentabilidad e inversión de los contribuyentes en la provincia de Chimborazo, Ecuador durante la pandemia de Covid-19 y su relación con el ciclo económico. Contempla el método inductivo y un nivel causal-explicativo, la recolección de datos fue a través de un cuestionario a una muestra de 307 contribuyentes de una población total efectiva de 39 503, donde el índice de Alfa de Cronbach es de $89.9 \%$. La selección de elementos muestrales fue en el marco del muestreo probabilístico aleatorio simple considerando como base de datos los contribuyentes del Servicio de Rentas Internas; el análisis de datos se realizó con el modelo de regresión logística multinomial y lineal. Los resultados muestran que una alta carga impositiva afecta significativamente en la liquidez, rentabilidad e inversión de los contribuyentes, y que las modificaciones de las tasas y el tipo de impuestos producen incrementos y reducciones en los ingresos, utilidades e inversiones. Se concluye que la presencia de un factor externo como el Covid-19 profundiza significativamente el efecto negativo en los ingresos y resultados financieros de la empresa y en futuras inversiones. Existe una relación directa entre el ciclo económico y las recaudaciones tributarias.
\end{abstract}

\begin{abstract}
High rates and different taxes determine tax collection in different economic cycles in economies; in this context the research analyses the effect of tax burden and pressure on liquidity, Profitability and investment of taxpayers in the Province of Chimborazo, Ecuador in times of the Covid-19 pandemic and its relationship to the economic cycle. It contemplates the inductive method and a causal level - explanatory, data collection was through the questionnaire to a sample of 307 contributors of a total effective population of 39 503, where the Cronbach Alpha index is $89.9 \%$. The selection of sample elements was in the framework of simple random probabilistic sampling considering as a database the contributors of the Internal Revenue Service; the data analysis was performed with the multinomial and linear logistic regression model. The results show that a high tax burden significantly affects taxpayers' liquidity, profitability and investment, and that changes in rates and rates lead to increases and reductions in income, profits and investments. It is concluded that the presence of an external factor such as COVID-19 significantly deepens the negative effect on the financial income and results of the company and on future investments. There is a direct relationship between the business cycle and tax revenue.
\end{abstract}

\section{Palabras clave I keywords}

Tributación, carga tributaria, liquidez, impuestos, rentabilidad, inversión, ingresos, ciclos económicos. Taxation, tax burden, liquidity, taxes, profitability, investment, income, business cycles.

Cómo citar: Quispe-Fernández, G. M., y Ayaviri-Nina, D. (2021). Carga y presión tributaria. Un estudio del efecto en la liquidez, rentabilidad e inversión de los contribuyentes en Ecuador. Retos Revista de Ciencias de la Administración y Economía, 11(22), pp. 251-270. https://doi.org/10.17163/ret. n22.2021.04 


\section{Introducción}

En América Latina, la carga y presión tributaria de los contribuyentes es alta por las diferentes tasas impositivas que soportan con respecto a sus ingresos, en el ámbito de país esto contribuye al desarrollo de las economías, las cuales son cíclicas en base a fases como la expansión, auge, crisis, recesión y depresión evidenciadas a través de los productos, la inversión y la rentabilidad de las empresas.

Las recaudaciones tributarias son una fuente de financiamiento para los ingresos nacionales y estos se relacionan con los ciclos económicos de las economías, las contribuciones dependen de los tipos de impuestos, a mayor carga y presión tributaria mayores serán los ingresos nacionales y del Estado; sin embargo, para los contribuyentes pueden tener efectos positivos o negativos relacionados con los ingresos, la rentabilidad y la inversión empresarial.

Por otro lado, desde la perspectiva contable tributaria de los contribuyentes, existe una relación entre la contabilidad comercial y fiscal, porque la contabilidad sirve de base para la determinación tributaria y presentar la deducción en los estados financieros a través de "la identificación, medición y síntesis de los hechos y de la realidad económica de una entidad" (Archel \& Goméz, 2014, pp. 103-104). De este modo se deduce que la contabilidad general y la tributaria no son independientes.

En ese contexto, se analizó la situación tributaria en el Ecuador en función de los ingresos tributarios para identificar razones del incremento o reducción de la carga y presión tributaria y determinar el efecto en la liquidez, rentabilidad e inversión de los contribuyentes fiscales y los ciclos económicos, considerando para ello el caso de la provincia de Chimborazo.

Los datos del Servicio de Rentas Internas (2020) muestran que la recaudación tributaria en el 2020 alcanzó alrededor de 11313 millones inferiores al 2019 que fue de 14268 millones de dólares, observándose una reducción en el 2019 y 2020 con relación al 2018; los ingresos tributarios alcanzan el 20,6 \% sobre el PIB (2018) (OCDE et al., 2020 , p. 60). Esta caída en la recaudación tributaria podría ser una consecuencia de la pandemia mundila de Covid-19 ocurrida a partir de marzo de 2019, teniendo su efecto en el ciclo económico del Ecuador (Tabla 1).

Asimismo, considerando que la carga tributaria:

No solo obedece a la legislación tributaria. También influyen en ella las normas no tributarias (el laboral, por ejemplo), la administración de impuestos y el cumplimiento de los contribuyentes, así como el nivel, la composición y la distribución de la actividad económica y el ingreso. (Rodríguez \& Ávila, 2017, p. 119)

Según datos de la tabla 1, la carga tributaria para el 2018 fue de $20.40 \%$ y en el 2019 el $20.1 \%$ (último dato), observándose un incremento desde el 2007 de $4.4 \%$ hasta 2019. Por otra parte, la presión fiscal que soportan los contribuyentes provenientes del Gobierno Central en el 2019 alcanzó $13.6 \%$, gobiernos subnacionales $1 \%$ y de la contribución a la seguridad social $5.2 \%$, deduciendo que existe mayor presión fiscal en la seguridad social e impuestos. Por lo tanto, el incremento en la carga tributaria puede "limitar significativamente las posibilidades de inversión y creación de empresas con tendencia sostenible en el largo plazo" (Sarmiento, 2010, p. 202), como también reducir los ingresos. 


\section{Tabla 1. Ecuador. Evolución de la recaudación y presión tributaria bruta (2000 a 2019) (Expresado en millones de dólares)}

\begin{tabular}{|c|c|c|c|c|c|c|c|}
\hline Años & $\begin{array}{l}\text { Recaudación } \\
\text { tributaria }\end{array}$ & $\begin{array}{l}\text { Ingresos } \\
\text { fiscales } \\
\text { (Per cápita) }\end{array}$ & $\begin{array}{c}\text { Ingresos } \\
\text { fiscales } \\
\text { (SUS) }\end{array}$ & $\begin{array}{l}\text { Presión } \\
\text { fiscal } \\
\text { (\% PIB) }\end{array}$ & $\begin{array}{c}\text { Presión } \\
\text { fiscal } \\
\text { Gobierno } \\
\text { Central }\end{array}$ & $\begin{array}{c}\text { Presión } \\
\text { fiscal } \\
\text { Gobiernos } \\
\text { Sub } \\
\text { nacionales }\end{array}$ & $\begin{array}{l}\text { Contribuciones } \\
\text { a la } \\
\text { Seguridad } \\
\text { Social }\end{array}$ \\
\hline 1999 & & 147.00 & 1819.40 & & & & \\
\hline 2000 & 1675.00 & 184.00 & 2300.80 & & & & \\
\hline 2001 & 2380.00 & 279.00 & 3573.80 & & & & \\
\hline 2002 & 2759.00 & 320.00 & 4184.50 & & & & \\
\hline 2003 & 2975.00 & 284.00 & 3778.60 & & & & \\
\hline 2004 & 3349.00 & 287.00 & 3892.20 & & 10.4 & 0.7 & 2.8 \\
\hline 2005 & 4046.00 & 334.00 & 4579.80 & & 10.9 & 0.7 & 2.7 \\
\hline 2006 & 4686.00 & 387.00 & 5407.40 & & 11.2 & 0.6 & 3.3 \\
\hline 2007 & 5344.00 & 395.00 & 5617.40 & 15.66 & 11.7 & 0.6 & 3.5 \\
\hline 2008 & 6409.00 & 432.00 & 6255.70 & 15.83 & 11.5 & 0.7 & 3.4 \\
\hline 2009 & 6890.00 & 477.00 & 7027.00 & 16.56 & 12.4 & 0.8 & 3.3 \\
\hline 2010 & 8070.00 & 559.00 & 8397.80 & 17.39 & 13.1 & 0.7 & 3.7 \\
\hline 2011 & 8894.00 & 658.00 & 10042.90 & 18.66 & 12.5 & 0.7 & 5.0 \\
\hline 2012 & 11216.00 & 868.00 & 13471.40 & 20.66 & 14.1 & 0.8 & 5.4 \\
\hline 2013 & 12638.00 & 887.00 & 13999.50 & 20.54 & 14.6 & 0.8 & 4.8 \\
\hline 2014 & 13523.00 & 919.00 & 14736.30 & 20.27 & 14.4 & 0.8 & 4.6 \\
\hline 2015 & 14341.00 & 1171.00 & 19059.70 & 21.96 & 15.9 & 1.0 & 5.1 \\
\hline 2016 & 13388.00 & 1087.00 & 17962.50 & 19.83 & 14.2 & 0.9 & 4.7 \\
\hline 2017 & 13680.00 & 1095.00 & 18363.70 & 19.82 & 13.7 & 0.9 & 5.2 \\
\hline 2018 & 15145.00 & 1106.00 & 18830.20 & 20.40 & 14.6 & 1.0 & 5.2 \\
\hline 2019 & 14268.00 & 1125.00 & 19422.70 & 20.10 & 13.6 & 1.00 & 5.50 \\
\hline 2020 & 13313.00 & & & & & & \\
\hline Promedio & 8523.29 & 590.95 & 9214.70 & & 13.92 & 0.86 & 4.54 \\
\hline
\end{tabular}

Fuente: SRI (2020a), Landázuri (2019).

Los contribuyentes con mayor participación empresarial es el sector de la microempresa (último dato) con $90.81 \%$, pequeña empresa $7.13 \%$, Mediana Empresa "A" $0.95 \%$, Mediana empresa “B” $0.64 \%$ y Grande empresa $0.47 \%$; donde el $42.66 \%$ son empresas de servicio y el $34.93 \%$ comercio, Agricultura, ganadería, silvicultura y pesca $10.38 \%$, Industrias Manufactureras $8.38 \%$, Construcción 3.43\%, Explotación de Minas y Canteras $0.22 \%$ (Directorio de Empresas- DIEE, 2018, en INEC, 2020). Por otro lado, en promedio las empresas cierran entre el tercero y quinto año de existencia, lo que influye en la recaudación tributaria (Alcivar \& Saines, 2011), es decir:

Las posibilidades de supervivencia para las microempresas alcanza el $52.7 \%$ y en las pequeñas empresas el $32.1 \%$ y en las medianas empresas A un $25.8 \%$, Medianas empresas un 25 $\%$ y las grandes empresas un $17.9 \%$, siendo la relación inversa entre el tamaño y la natali$\mathrm{dad} / \mathrm{mortalidad}$ de las empresas. (INE, 2017, p. 64). 
La tasa de entrada durante el 2010 al 2015 en el sector de la manufactura fue de $20.66 \%$ y la salida el $11.57 \%$, relativamente menor al resto de los sectores, pero superior al $10 \%$ (tabla 2$)$.

Tabla 2. Entradas y salidas de Empresas (porcentaje) 2010-2015

\begin{tabular}{|l|r|r|r|}
\hline \multicolumn{1}{|c|}{ Sctor } & Tasa de entrada & Tasa de salida & \multicolumn{1}{c|}{ Diferencia } \\
\hline Construcción & 38.65 & 24.91 & 13.74 \\
\hline Minas & 37.07 & 18.13 & 18.94 \\
\hline Servicios & 28.94 & 17.12 & 11.82 \\
\hline Comercio & 24.16 & 11.64 & 12.52 \\
\hline Manufactura & 20.66 & 11.57 & 9.09 \\
\hline Promedio & 29.90 & 16.67 & 13.22 \\
\hline
\end{tabular}

Fuente: $\operatorname{INEC}$ ( 2017, p. 70)

Según datos del INEC (2020) las ventas totales en el 2018 (último dato disponible) en el sector comercio tiene la mayor participación con $38.08 \%$, seguido por servicios $(24.40 \%)$, manufactura $(21.43 \%)$, minería y canteras $(6.85 \%)$, agricultura, ganadería, silvicultura y pesca $(5.75 \%)$ y construcción $(3.49 \%)$; siendo las ventas mayores en grandes empresas $71.97 \%$, pequeñas empresas $11.29 \%$, medianas empresas B $9.65 \%$, medianas empresas A $6.15 \%$ y microempresas $0.93 \%$.

Por tanto, se deduce que los niveles de venta influyen en la liquidez empresarial. Sin embargo, la liquidez financiera puede estar sujeta a la carga tributaria o impositiva y presión fiscal; al ser alta la carga tributaria, un porcentaje de la liquidez podría ser destinada al pago de los tributos, esto podría afectar a la rentabilidad e inversión.

En cuanto a la rentabilidad, mencionan autores como Fernández (2004a) y Chen et al. (2010):

Cómo las compañías con mayor rentabilidad tendrán mayores incentivos para desplegar estrategias de reducción de sus cargas tributarias, mostrando una mayor divergencia entre tipos nominales y reales. Por último, el riesgo fiscal también podría explicar la conducta de las empresas en el orden tributario y tratarse de un elemento moderador de prácticas fiscales más agresivas. (Monterrey \& Sánchez, 2015 citado en Monterrey \& Sánchez, 2020, p.223)

Con estos antecedentes, la investigación pretendió responder a la pregunta ¿Cómo afecta una alta carga tributaria en la liquidez económica, la rentabilidad e inversión de los contribuyentes en el Ecuador en época de pandemia-Covid-19 y cuál es la relación con el ciclo económico?

Luego de una revisión bibliográfica, se observan diferentes estudios en distintos países, como Belloso (2010) analiza el impuesto a las transacciones; Sarmiento (2010) la carga fiscal; Pecho y Peragón (2013) y Quispe et al. (2019) evolución de las reformas tributarias; Crespo (2016) las distintas formas de calcular la carga impositiva; Monterrey y Sánchez (2017) la relación de la presión fiscal en la inversión; Salto et al. (2018) los beneficios de los tributos; Márquez et al. (2018) los efectos de las reformas tributarias en la recaudación; Monterrey y Sánchez (2020) la evolución de la presión fiscal; Rodríguez y Ávila (2017) distribución de la carga tributaria; Piedra et al. (2016) caracterizan a los contribuyentes; Cardoso y Funchal (2011) evalúan el efecto de la regulación laboral y tributaria; Llamas et al. (2019) miden el efecto del impuesto sobre la renta; Lima y Resende (2019) verifican los tributos que más contribuyen en la carga tributaria; Chávez y López (2019) analizan los factores que inciden en la recaudación 
inmobiliaria; Brito-Gaona e Iglesias (2017) evidencian sobre el incremento de los impuestos y el gasto público.

Es importante resaltar la investigación realizada por Sarmiento (2010, p. 204) quien menciona "que un nivel de carga fiscal incide desfavorablemente en la situación financiera”; en ese marco Crespo (2016) y Quispe et al. (2017) muestran los distintos impuestos que conforman la carga fiscal en el caso de Ecuador, y Monterrey y Sánchez (2017) plantean que "los pagos fiscales futuros son una motivación adicional para adoptar una inversión"; Márquez et al. (2018, p. 3) "evidencia la incidencia de la presión fiscal en los contribuyentes por los cambios impositivos"; Ruiz-Vargas y NavarroMorato (2016, p. 109) manifiestan que existe un efecto en el impuesto a la renta cuando se deduce la tarifa; y Brito-Gaona e Iglesias (2017) muestran que la presión tributaria "tiene efectos significativos en la inversión privada".

De este modo la investigación con los antecedentes prácticos y teóricos tuvo como objetivo determinar el efecto de la carga tributaria sobre la liquidez, rentabilidad e inversión de los contribuyentes en el Ecuador durante la pandemia Covid-19 y su relación con el ciclo económico, dado que la mayoría de los estudios fueron realizados antes de la pandemia y se considera la inversión externa en la mayoría de los estudios y muy poca inversión interna, de ahí su relevancia. Por esto se planteó como hipótesis general H1 las altas tasas, tipos, cambios y el Covid-19 inciden significativamente en los ingresos, utilidades e inversión de los contribuyentes fiscales y secundarias H1a. Las altas tasas impositivas influyen negativamente en los ingresos de los contribuyentes; $\mathrm{H} 1 \mathrm{~b}$ las altas tasas y varios tipos de impuestos tienen un efecto negativo en la liquidez, rentabilidad e inversión de los contribuyentes, porque reducen la inversión y la rentabilidad y afectan en la liquidez; H1c los cambios en los impuestos y las tasas generan reducciones o incrementos en los ingresos, utilidades e inversión en función al grado de significancia del tipo de impuesto; H1d la presencia de un factor externo como el Covid-19 influye significativamente en los ingresos; y H2 existe una relación directa entre el ciclo económico y el ciclo de la recaudación tributaria.

\subsection{Aspectos teóricos}

La investigación comprende que un contribuyente "es la persona natural o jurídica a quien la ley impone la prestación tributaria por la verificación del hecho generador" (Congreso Nacional, 2018, Art. 25).

El Decreto Ejecutivo 1021 publicado bajo Registro Oficial $\mathrm{N}^{\circ} 173$ del 31 de marzo 2020, reforma el Reglamento para la Aplicación de la Ley de Régimen Tributario Interno (LORTI) D.E. 374, R.O 209 del 8 de junio del 2010, define una retención mensual sobre el total de ingresos gravados. La Ley Orgánica para la Reactivación de la Economía, Fortalecimiento de la Dolarización y Modernización de la Gestión Financiera (2017) R.O.1 N 150 del 29 de diciembre de 2017, menciona que existe dos tipos de contribuyentes:

1) Personas naturales: a) obligadas a llevar contabilidad (ingresos mayores a dólares 100000 o capital de trabajo mayor a dólares 60000 y Costos y gastos anuales superiores a dólares 80 000 (Art. 37) y b) contribuyentes obligados a llevar cuentas de ingreso y egresos (Art. 38). 2) Personas jurídicas: sociedades. (Asamblea Nacional del Ecuador, 2018, Art. 98)

Respecto a las personas jurídicas la ley obliga a los contribuyentes a llevar contabilidad y pagar un conjunto de impuestos dependiendo del tipo de actividad; por ejemplo; Impuesto a las sociedades (22\%), Impuesto a la Renta (25\%) del total de ingresos gravables y sujeto a reducción del $10 \%$ por reinversión y $15 \%$ por distribución de utilidades); Impuesto a la salida de divisas (5\%); Impuesto al valor agregado 
(12\%); Impuesto sobre propiedades o prediales; Impuesto municipal sobre activos totales $(0.12 \%)$, entre otros.

Desde esa perspectiva, la liquidez es "la capacidad de la empresa para generar recursos que le permita atender con ellos sus compromisos a corto plazo” (Díaz, 2012, p.139).

En el artículo 98 del Código Tributario del 2018 del Ecuador liquidez es "el grado en que una empresa puede hacer frente a sus obligaciones corrientes, es la medida de su liquidez a corto plazo" y "que la liquidez está referida a la solvencia de la posición financiera en general de la organización, lo que se traduce en la facilidad que tiene la empresa para pagar sus deudas" (Nava, 2009, p. 613).

La rentabilidad "es la relación que existe entre la utilidad y la inversión necesaria para lograrla” (Zamora 2008, p. 57), es "un coeficiente que mide la utilidad generada por una inversión" (Parada 1988, p.15). De este modo, las "Decisiones de inversión están basadas en la rentabilidad esperada” (Monterrey \& Sánchez, 2020, p.198).

Roca et al. (2004, p. 33) mencionan que el impacto o efectos de la carga tributaria puede medirse de cuatro formas: "i) equilibrio del mercado, ii) la rentabilidad de las empresas formales e informales del sector, iii) la recaudación fiscal, y iv) el presupuesto y bienestar de los usuarios". El impacto sobre la rentabilidad de las empresas, consiste "en estimar sobre la estructura de costos, a través de los flujos de caja y el estado de pérdidas y ganancias” (Roca et al., 2004, p. 46) y el impacto sobre la recaudación fiscal, supone "considerar los dos modelos tanto de costeo como del análisis de incidencia tributaria" (Roca et al., 2004, p. 46).

Con este antecedente, la investigación consideró medir el impacto a través de los efectos de la carga tributaria en la liquidez, rentabilidad e inversión de las empresas de forma cualitativa y cuantitativa, dado que el efecto es una cadena que comienza en las reformas tributarias promulgadas por los distintos gobiernos, donde se modifican los impuestos y sus tasas, que traería como consecuencia variaciones en los flujos de ingreso, utilidades, rentabilidad, inversiones y en los precios, la fuerza laboral y finalmente en la economía.

\section{Metodología}

Se utilizó el método inductivo y tiene un nivel causal-explicativo. Se realizó una encuesta a 381 contribuyentes que corresponde al tamaño de la muestra, de los cuales se validaron 307 , la diferencia corresponde a cuestionarios no completados en su totalidad que se consideran como casos perdidos, siendo tratados estadísticamente las 307 encuestas válidas. La selección de los sujetos fue aleatoria considerando la base de datos de contribuyentes del SRI con corte al 2019 y una estratificación zonal a través de parroquias urbanas (tabla 3 ).

\section{Tabla 3. Población y muestra}

\begin{tabular}{|c|c|c|c|c|c|c|}
\hline 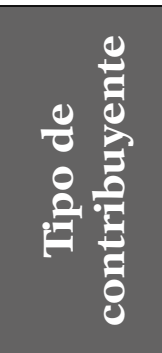 & 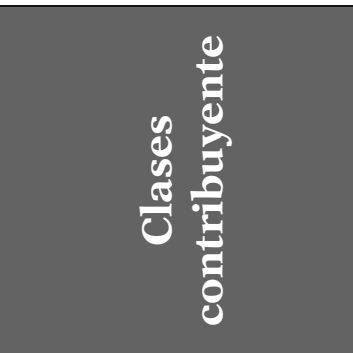 & 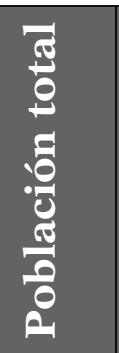 & 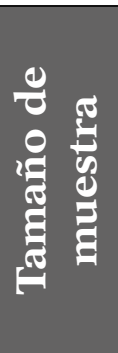 & 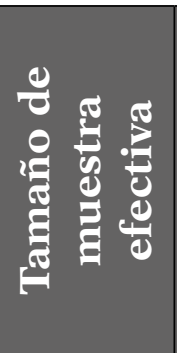 & 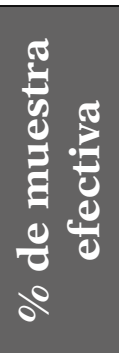 & $\frac{\mathscr{8}}{8}$ \\
\hline \multirow{2}{*}{ Especial } & Sociedades & 374 & 4 & 5 & 2 & Parroquia Lizarzaburo \\
\hline & Personas naturales & 46 & 0 & 0 & 0 & Parroquia Veloz \\
\hline
\end{tabular}




\begin{tabular}{|l|l|r|r|r|r|l|}
\hline \multirow{2}{*}{ Otros } & Sociedades & 2023 & 20 & 1 & 0 & Parroquia Maldonado \\
\cline { 2 - 6 } & Personas naturales & 27256 & 262 & 175 & 57 & Parroquia Velasco \\
\hline RISE & Personas naturales & 9804 & 95 & 126 & 41 & Parroquia Maldonado Centro \\
\hline Total & Total & 39503 & 381 & 307 & 100 & Total \\
\hline
\end{tabular}

Fuente: Elaboración propia.

Se recogió información en distintas zonas parroquiales; la elaboración del cuestionario responde a la operacionalización de variables; el cuestionario consideró escalas de medidas de tipo likert de 5 puntos, tiene un índice de Alfa de Cronbach global de $0.899(89.9 \%)$ que corresponde a una validación interna o de contenido, considerando que "los valores son aceptables cuando son iguales o superiores a 0,70 y menores o iguales a 0,90" (Campo-Arias \& Oviedo, 2008, p. 837); y externa o de criterio a través de una prueba piloto realizada a 20 contribuyentes expertos y dos especialistas en tributación, su análisis permitió mejoras en las preguntas. La consistencia de cada sección del cuestionario se presenta en la tabla 4.

Tabla 4. Consistencia del cuestionario por secciones

\begin{tabular}{|l|r|r|r|}
\hline \multicolumn{1}{|c|}{ Preguntas } & $\begin{array}{c}\text { Alfa de } \\
\text { Cronbach }\end{array}$ & $\begin{array}{c}\text { Alfa de Cronbach } \\
\text { basada en elementos } \\
\text { estandarizados }\end{array}$ & $\begin{array}{c}\text { No de } \\
\text { elementos }\end{array}$ \\
\hline Caracterización de los contribuyentes & 0.834 & 0.781 & 12 \\
\hline Elementos contables & 0.687 & 0.695 & 6 \\
\hline Tipos de Impuestos & 0.897 & 0.900 & 23 \\
\hline Ingresos, gastos, ahorro e inversión & 0.806 & 0.816 & 8 \\
\hline Destino de los ahorros & 0.747 & 0.746 & 7 \\
\hline $\begin{array}{l}\text { Motivación para cumplimiento } \\
\text { tributario }\end{array}$ & 0.829 & 0.829 & 11 \\
\hline Causas & 0.872 & 0.872 & 11 \\
\hline Efectos & 0.883 & 0.883 & 13 \\
\hline Cultura tributaria & 0.823 & 0.823 & 13 \\
\hline $\begin{array}{l}\text { Valores cuantitativos sobre venta, gas- } \\
\text { tos, utilidades, pago de impuestos }\end{array}$ & 0.609 & 0.843 & 7 \\
\hline
\end{tabular}

Fuente: Elaboración propia.

Para el análisis y la demostración de las hipótesis, se aplicaron modelos de regresión considerando que los efectos se relacionan con: La riqueza, los ingresos y el consumo; en cuanto a los tipos de tasas, se considera dos: los servicios públicos y las tasas fiscales, donde las contribuciones pueden ser de forma directa o indirecta, y finalmente con relación a su incidencia se debe considerar a las empresas desde el punto de vista de su carga tributaria y presión tributaria, dando lugar no solo al efecto económico, sino también a lo legal de los impuestos (Sarmiento, 2010).

De este modo se utilizó el modelo de regresión logística multinomial para datos cualitativos y regresión lineal para cuantitativos. 


\section{Resultados}

\subsection{Caracterización y concentración geográficas de los contribuyentes}

La ciudad de Riobamba en la provincia Chimborazo, Ecuador, tiene una población según el último censo poblacional de 2010 (INEC, 2020) de 234170 habitantes lo que representa el $49 \%$ del total de población de la provincia de Chimborazo, con una proyección para el 2020 de 264048 habitantes que llegaría a representar el $50 \%$.

Existen alrededor de 39503 contribuyentes activos para el 2020 (dato que puede cambiar en función a la actualización del SRI) ubicados en las distintas zonas parroquiales de la ciudad de Riobamba que desarrollan distintas actividades económicas en el ámbito local, provincial y nacional. Estos tienen sus plantas de producción, transformación y comercialización localizadas en cinco parroquias urbanas: parroquia Maldonado, parroquia Lizarzaburo, parroquia Velasco, parroquia Veloz y parroquia Yaruquies. La investigación revela que existe una mayor concentración de los contribuyentes en la parroquia Lizarzaburo en un $40.4 \%$, seguida de la parroquia Velasco con el $23.8 \%$, parroquia Maldonado con $18.9 \%$, parroquia Veloz con $14.7 \%$ y parroquia Yaruquies con el $2.3 \%$ (gráfico 1).

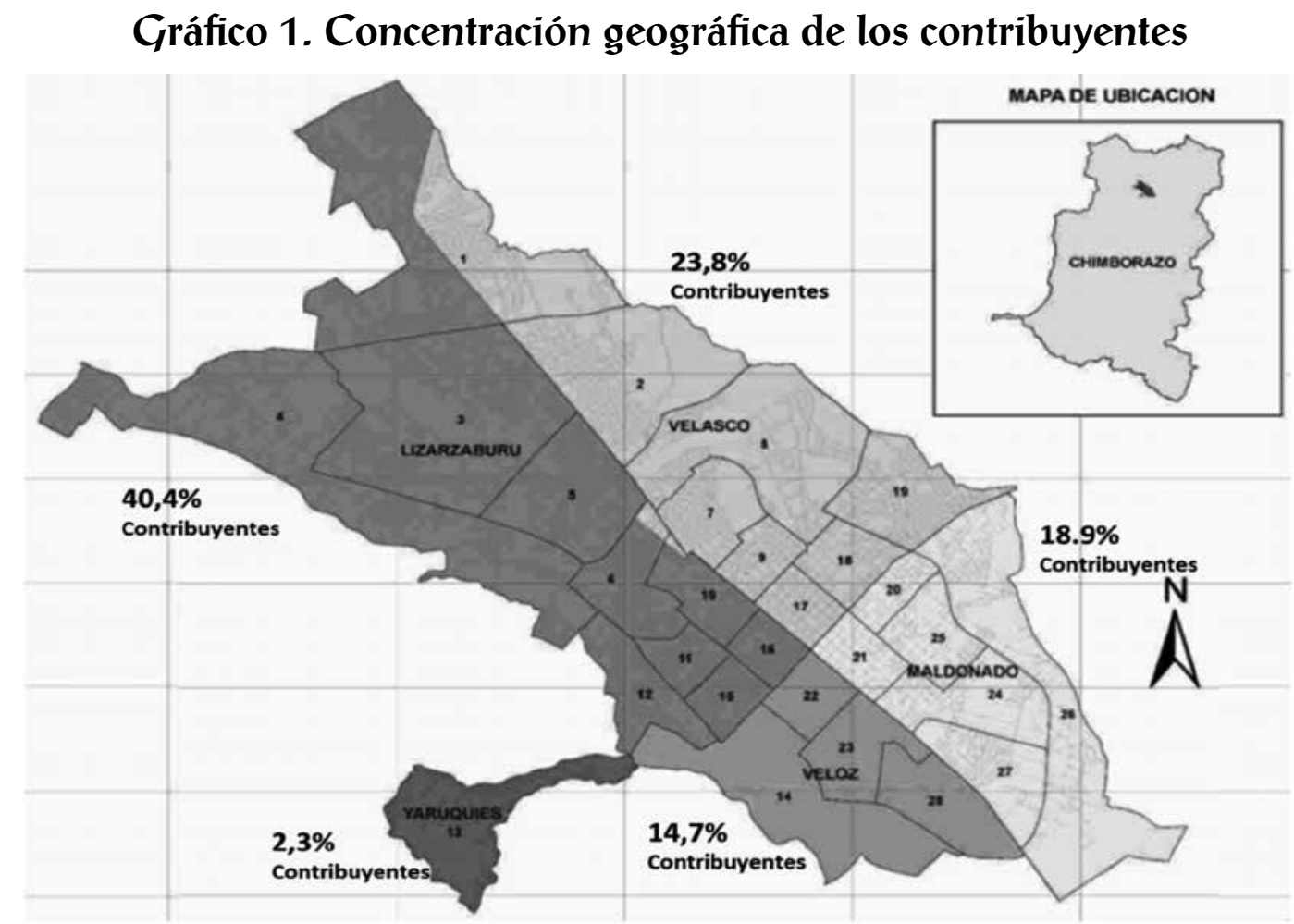

Fuente: Elaboración propia.

\subsection{Participación de los contribuyentes en las actividades económicas}

El comercio es la actividad con mayor participación $46.9 \%$, seguido por servicios $35.8 \%$, construcciones $8.1 \%$; cooperativas $5.9 \%$, industria manufacturera $2 \%$; explotación de minas y canteras $0.7 \%$; agricultura, ganadería, silvicultura y pesca $0.7 \%$ (tabla 8 ).

\subsection{Clasificación de los contribuyentes}

Los resultados muestran que los contribuyentes clasificados por tipo de constitución jurídica, $53.19 \%$ son individuales; $28.76 \%$ sociedad; $17.26 \%$ familiar y el $0.98 \%$ 
otros. Asimismo, el $41.04 \%$ pertenecen al Régimen Impositivo Simplificado (RISE); $57 \%$ Régimen General (RG); 1,63\% Especial y $0.33 \%$ otros. Al realizar un cruce de variables: 1) los contribuyentes individuales en un $54.6 \%$ pertenecen al RISE, $44.8 \%$ RG, $0.6 \%$ especial; 2 ) los de sociedad, $14.6 \%$ RISE, $85.2 \%$ RG, $1.1 \%$ especial, y 3) los familiares, $47.2 \%$ pertenece al RISE, 47.2 \% RG y $5.7 \%$ especial. Deduciendo que los contribuyentes RISE en su mayoría son individuales (70.6\%); en el Régimen General $(47.2 \%)$ y Especiales son familiares $(60 \%)$.

\subsection{Los componentes de la carga impositiva y su relación con los ingre- sos de los contribuyentes}

La carga impositiva permite:

Estimar el peso relativo de cada ítem sobre las ventas o ingresos totales, luego ponderar, individualmente, por las correspondientes tasas tributarias a las que esta afecta, con lo que finalmente se obtiene la incidencia tributaria neta como porcentaje de los ingresos totales. (Roca et al., 2004, p. 23)

Los resultados, muestran que la carga impositiva está compuesta por 23 tipos de impuestos, siendo las más significativas: el Impuesto a la Renta (78.2\%), Impuesto al Valor Agregado (73\%), Impuestos de Patentes Municipales (57.7\%), Impuesto al Predio Urbano $(47.9 \%$ ) (gráfico 2$)$.

\section{Gráfico 2. Carga impositiva de los contribuyentes (Expresado en porcentaje)}

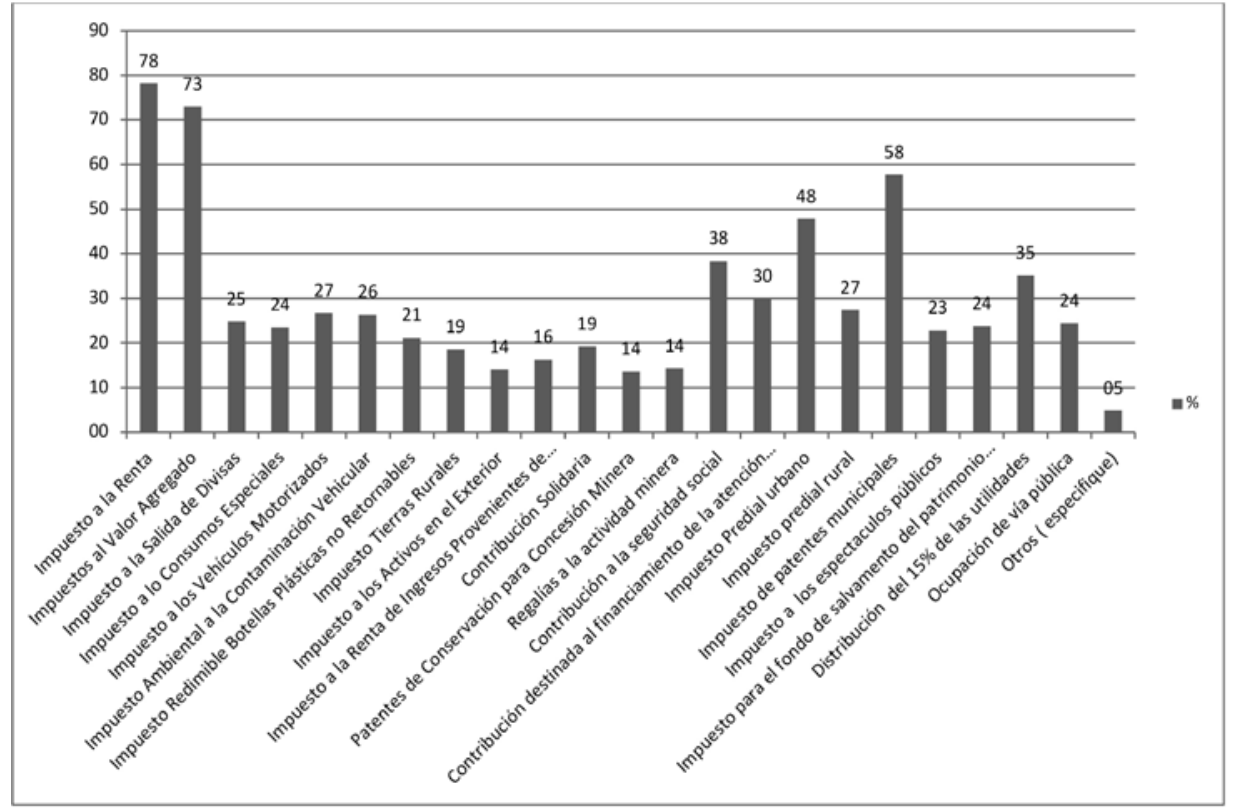

Fuente: Elaboración propia.

Asimismo, los ingresos promedios mensuales de los contribuyentes alcanzan una media de \$24 329 al mes y \$149 457.87 al año; en el 2020 fueron afectados por la pandemia, provocando disolución o liquidación de las empresas. Los datos muestran que al rededor del $0.7 \%$ de las empresas inscritas en la Superintendencia de Compañias se disolvieron o liquidaron (Superintendencia de Compañias, 2020). Consultando a los contribuyentes, el $47.7 \%$ está de acuerdo con que las tasas de los impuestos son altas, el $45.6 \%$ existen varios tipos de impuestos y el $44 \%$ afecta a los ingresos (tabla 5). 


\section{Tabla 5. Variables relacionadas a los impuestos e ingresos de los contribuyentes (porcentaje)}

\begin{tabular}{|c|c|c|c|c|c|c|}
\hline$\frac{d}{\frac{d}{0}}$ & 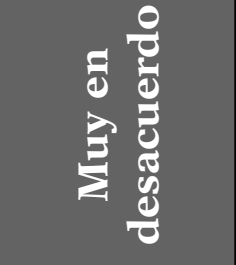 & 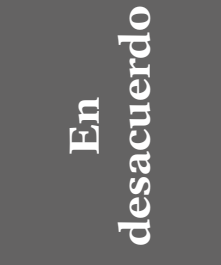 & 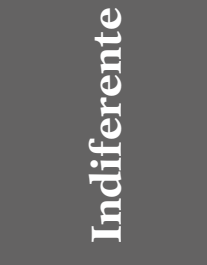 & 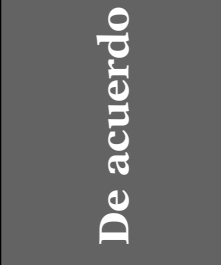 & 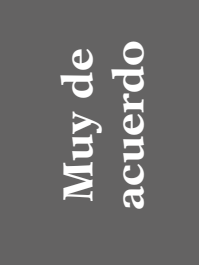 & हृ \\
\hline $\begin{array}{l}\text { V1. Altas tasas } \\
\text { de los impues- } \\
\text { tos }\end{array}$ & $4.20 \%$ & $10.10 \%$ & $22.10 \%$ & $47.60 \%$ & $16.00 \%$ & $100.00 \%$ \\
\hline $\begin{array}{l}\text { V2. Varios tipos } \\
\text { de impuestos a } \\
\text { pagar }\end{array}$ & $2.00 \%$ & $10.10 \%$ & $27.60 \%$ & $45.60 \%$ & $14.70 \%$ & $100.00 \%$ \\
\hline $\begin{array}{l}\text { V3. Afecta a los } \\
\text { ingresos de la } \\
\text { empresa }\end{array}$ & $5.20 \%$ & $7.50 \%$ & $26.10 \%$ & $44.00 \%$ & $17.30 \%$ & $100.00 \%$ \\
\hline $\begin{array}{l}\text { Modelo } 1 \text { a. } \\
\text { Variable depen- } \\
\text { diente: Afecta } \\
\text { el ingreso de la } \\
\text { empresa } \\
\text { b. Predictores: } \\
\text { V1 alta tasas de } \\
\text { impuesto }\end{array}$ & $\begin{array}{l}\text { R cuadrado } \\
0.339\end{array}$ & $\begin{array}{l}\text { Ajuste } \\
\text { Sig. } 0.000\end{array}$ & $\begin{array}{l}\text { Desviación } \\
0.000\end{array}$ & $\begin{array}{l}\text { Aporte a la } \\
\text { explicación } \\
\text { De acuerdo } \\
73.30 \% \\
\text { Beta } 2.836 \\
\text { Sig. } 0.000\end{array}$ & $\begin{array}{l}\text { Aporte a } \\
\text { la explica- } \\
\text { ción muy } \\
\text { de acuer- } \\
\text { do } 58.50 \\
\%\end{array}$ & $\begin{array}{l}\text { Aporte a la } \\
\text { explicación } \\
\text { indiferente } \\
40.00 \% \\
\text { Beta } 2.923 \\
\text { Sig. } 0.000\end{array}$ \\
\hline
\end{tabular}

Fuente: Elaboración propia.

El modelo de regresión logística polinomial aplicado a las variables categóricas, los resultados permiten demostrar que H1a las altas tasas impositivas (presión fiscal) afecta significativamente en los ingresos de los contribuyentes en $33.9 \%$ (Dependencia Sig. 0,000 ) profundizándose en el 2020 como consecuencia de las restricciones por la pandemia de Covid-19. Además, un incremento de las tasas afectaría en una reducción de 0.257 en los ingresos y un incremento de número total de impuestos afectaría en la reducción de ingresos en 0.542 , por lo que la carga tributaria afecta negativamente a los contribuyentes.

\subsection{Medición cualitativa del efecto de la carga y presión tributaria en la liquidez, rentabilidad e inversión}

La aplicación del modelo de regresión logística multinomial a variables independientes: altas tasas y tipos de impuestos; variables dependientes: ingreso Modelo 1, utilidad Modelo 2, inversión Modelo 3. Los resultados del Modelo 1 muestran que las altas tasas afectan a los ingresos en $33.9 \%\left(\mathrm{R}^{2}=0.339\right.$ Sig. 0.000$)$. En el Modelo 2 afecta en la reducción de utilidades en $30.2 \%\left(\mathrm{R}^{2}=0.302\right.$ Sig. 0.001$)$ con una relación del $55 \%$ $(\mathrm{R}=0.550)$. En el Modelo 3 afecta en la reducción de la inversión en $28.9 \%\left(\mathrm{R}^{2}=0.289\right.$ Sig.0.000) con una relación del $44.1 \%(\mathrm{R}=0.441)$ (tabla 6). Esto significa que H1b las altas tasas y varios tipos de impuestos tienen un efecto negativo en la liquidez, rentabilidad e inversión de los contribuyentes es cierta, porque reducen la inversión y la rentabilidad y afectan en la liquidez; además el contribuyente tiene la obligación de pagar un conjunto de impuestos y no recibir estímulos fiscales que generen mejorar la rentabilidad; como mencionan Hall y Dale (1967) existe una efectividad de los incentivos 
fiscales en la inversión, porque una deducción de las tasas de impuestos por inversión puede estimular el incremento de inversiones en activos. Por lo tanto, el incremento de la carga tributaria en los contribuyentes desestimula la inversión, afecta a la liquidez y la rentabilidad y a la dinámica del desarrollo empresarial, agudizando la crisis económica en un país afectado por la pandemia de Covid-19.

Tabla 6. Resultados del modelo de regresión

\begin{tabular}{|c|c|c|c|c|c|}
\hline Modelo & $\begin{array}{c}\text { R } \\
\text { cuadrado }\end{array}$ & Sig. & Pearson & $\begin{array}{c}\text { Altas tasas } \\
\text { Indiferente }\end{array}$ & $\begin{array}{c}\text { Tipos } \\
\text { impuestos } \\
\text { De acuerdo }\end{array}$ \\
\hline $\begin{array}{c}\text { Modelo 1. (Y1) Afecta } \\
\text { a ingresos }\end{array}$ & 0.339 & 0.000 & 0.000 & $\begin{array}{c}\text { Beta } 2.836 \\
\text { Sig. } 0.000\end{array}$ & $\begin{array}{c}\text { Beta } 2.923 \\
\text { Sig. } 0.000\end{array}$ \\
\hline $\begin{array}{c}\text { Modelo 2. (Y2) Reduc- } \\
\text { ción en utilidades }\end{array}$ & 0.302 & $0.000^{\mathrm{b}}$ & 0.550 & $\begin{array}{c}\text { Beta } 2.808 \\
\text { Sig. } 0.001\end{array}$ & $\begin{array}{c}\text { Beta } 1.800 \\
\text { Sig. } 0.001\end{array}$ \\
\hline $\begin{array}{c}\text { Modelo 3. (Y3) Reduc- } \\
\text { ción en inversión }\end{array}$ & 0.289 & 0.000 & 0.441 & $\begin{array}{c}\text { Beta } 3.670 \\
\text { Sig. } 0.000\end{array}$ & $\begin{array}{c}\text { Beta } 1.574 \\
\text { Sig. } 0.000\end{array}$ \\
\hline
\end{tabular}

Fuente: Elaboración propia.

\subsection{Medición cuantitativa de la carga tributaria}

La aplicación de la regresión lineal a datos cuantitativos de las variables: muestra que la carga tributaria afecta significativamente al ingreso en $93.1 \%\left(\mathrm{R}^{2}=0.931\right.$, $\mathrm{R}=0.965$, Sig. 0.000); liquidez financiera $92.6 \%\left(\mathrm{R}^{2}=0.926, \mathrm{R}=0.962\right.$, Sig. 0.000); y rentabilidad en $91.2 \%\left(\mathrm{R}^{2}=0.912, \mathrm{R}=0.955\right.$, Sig. 0.000); y no a la inversión (0\%) (tabla 7).

Tabla 7. Medición cuantitativa del impacto de la carga tributaria

\begin{tabular}{|l|l|l|l|l|}
\hline \multicolumn{2}{|c|}{ Modelo } & \multicolumn{1}{c|}{ Resumen } \\
\hline Ingresos & $0.965^{\mathrm{a}}$ & 0.93 & \multicolumn{1}{c|}{$\begin{array}{c}\text { R cuadrado } \\
\text { ajustado }\end{array}$} & \multicolumn{1}{c|}{ Sig. } \\
\hline Liquidez & $0.962^{\mathrm{a}}$ & 0.93 & 0.93 & $0.000^{\mathrm{b}}$ \\
\hline Rentabilidad & $0.955^{\mathrm{a}}$ & 0.91 & 0.93 & $0.000^{\mathrm{b}}$ \\
\hline Inversión & $0.001^{\mathrm{a}}$ & 0.00 & 0.91 & $0.000^{\mathrm{b}}$ \\
\hline $\begin{array}{l}\text { a. Predictores: (Constante), ¿A cuánto asciende el pago de impuesto de la empresa? } \\
\text { Anual (dólares) }\end{array}$ & \multicolumn{5}{l}{} \\
\hline
\end{tabular}

Fuente: Elaboración propia.

Significa que una alta carga tributaria tiene un efecto negativo significativo en la liquidez y rentabilidad de los contribuyentes, como no ocurre con la inversión, y esto se agudiza cuando existe una pandemia, siendo cierta la H1b.

\subsection{Efectos de los impuestos y las tasas}

Se identifica que H1c los cambios en los impuestos y las tasas generan reducciones o incrementos en los ingresos, utilidades e inversión en función al grado de significancia del tipo de impuesto (tabla 8), donde: un incremento en el impuesto a la renta puede reducir la inversión en un $19.8 \%$, las utilidades un $1.7 \%$; sin embar- 
go, no tendría un efecto en los ingresos, ya que no es un determinante significativo (Sig. 0.823); en cambio la reducción del Impuesto al Valor Agregado incrementaría la inversión $(15.9 \%)$, las utilidades $(4.8 \%)$ y los ingresos $(3.7 \%)$ aunque la utilidad y los ingresos no depende del IVA. Las contribuciones solidarias afectarían reduciendo la inversión $(25.0 \%$ ) y las utilidades (19.4) de manera significativa, como las contribuciones destinadas a la financiación integral del cáncer, reduciría las utilidades en $15.5 \%$ y el Impuesto para el fondo de salvamento del patrimonio afectaría en los ingresos en un $14.6 \%$. También, se observa que un incremento en las patentes de conservación para la concesión minera incrementaría los ingresos de manera significativa en un $15.4 \%$, del mismo modo el Impuesto a las patentes aumentaría las utilidades (14.9\%) y los ingresos (12.9\%); y el pago por la ocupación de vía pública incrementaria en un $13,7 \%$. Finalmente un incremento en las tasas de los impuestos reduciría la inversión en $14.2 \%$, las utilidades en un $18.9 \%$ y afectaría a los ingresos en un $47.8 \%$. 


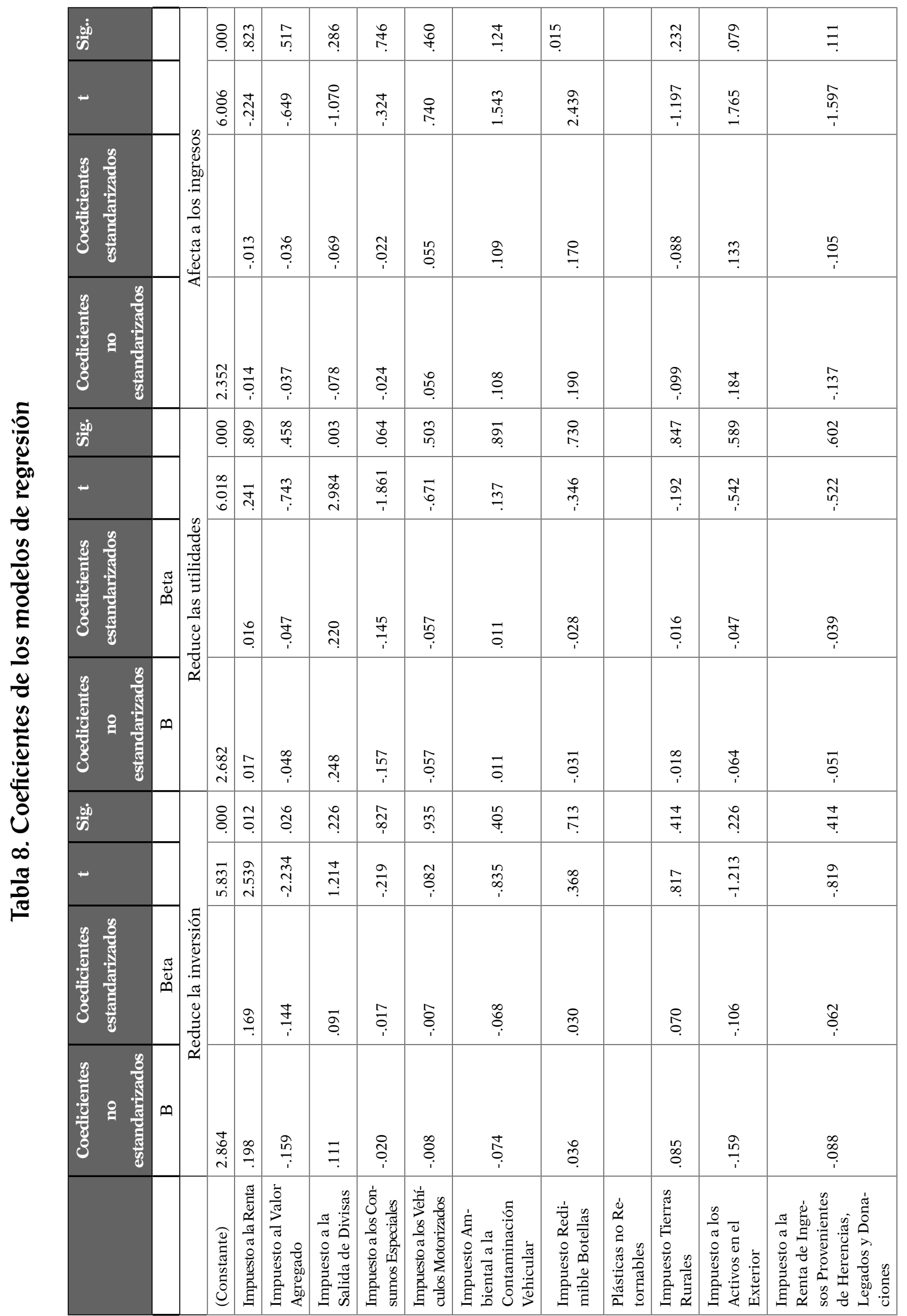




\begin{tabular}{|c|c|c|c|c|c|c|c|c|c|c|c|}
\hline$\underset{\infty}{\mathfrak{n}}$ & 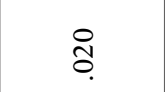 & $\overrightarrow{\tilde{n}}$ & $\stackrel{\odot}{?}$ & $\stackrel{\Xi}{\exists}$ & 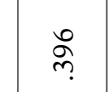 & 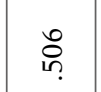 & $\underset{ }{\stackrel{丶}{f}}$ & के & 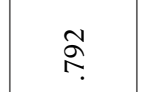 & డ̊. & \& \\
\hline$\stackrel{n}{n}$ & 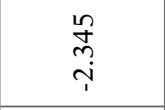 & 声 & $\stackrel{\text { of }}{\text { to }}$ & 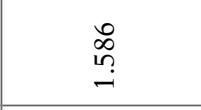 & 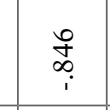 & : & $\stackrel{\Re}{\rightleftharpoons}$ & $\underset{\mathrm{i}}{\overrightarrow{\mathrm{f}}}$ & ঙ্ণ & $\begin{array}{l}\overrightarrow{\widetilde{n}} \\
\underset{i}{i}\end{array}$ & 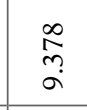 \\
\hline $\bar{\sigma}$ & 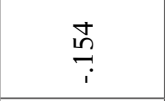 & ్ֶ & $\stackrel{n}{\exists}$ & $\stackrel{\square}{\varphi}$ & $\stackrel{\substack{n \\
i}}{i}$ & $\underset{+}{+}$ & $\underset{i}{\tilde{i}}$ & $\stackrel{0}{+}$ & ڤి & $\stackrel{\hat{m}}{i}$ & $\vec{F}$ \\
\hline$\cong$ & जิ & $\tilde{o}_{\hat{i}}^{\tilde{i}}$ & $\stackrel{\infty}{\stackrel{\circ}{\hookrightarrow}}$ & $\stackrel{m}{a}$ & $\underset{\text { ơ }}{o}$ & tơ & $\hat{\sigma}$ & $\stackrel{\infty}{+}$ & $\stackrel{\partial}{\partial}$ & $\stackrel{\text { fo }}{i}$ & \begin{tabular}{l}
$\infty$ \\
\multirow{f}{*}{}
\end{tabular} \\
\hline$\hat{\sigma}$ & 怘 & 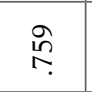 & $\stackrel{\stackrel{\partial}{\infty}}{\infty}$ & $\tilde{o}$ & ğ & $\stackrel{\tilde{m}}{?}$ & $\stackrel{m}{m}$ & $\stackrel{\infty}{\stackrel{\infty}{\uparrow}}$ & 。․ & $\stackrel{\bar{\phi}}{\bar{\infty}}$ & $\overline{\mathrm{o}}$ \\
\hline 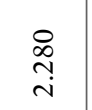 & $\overrightarrow{\mathcal{f}}$ & ô & స్తి & $\underset{i}{\stackrel{O}{i}}$ & $\tilde{E}$ & 导 & 吕 & $\stackrel{\leftrightarrow}{\stackrel{8}{\circ}}$ & 寿 & $\overrightarrow{\widetilde{r}}$ & 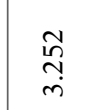 \\
\hline$\stackrel{\infty}{=}$ & $\tilde{\tilde{c}}$ & ڤ్ & $\stackrel{\infty}{0}$ & $\stackrel{n}{\leftrightarrow}$ & $\stackrel{\circ}{8}$ & $\stackrel{m}{i}$ & $\stackrel{\circ}{\circ}$ & $\stackrel{1}{\infty} \stackrel{0}{a}$ & 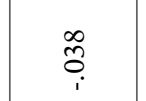 & $\stackrel{\circ}{0}$ & $\stackrel{\infty}{\hookrightarrow}$ \\
\hline$\stackrel{t}{\stackrel{+}{\prime}}$ & $\underset{i}{*}$ & $\hat{o}_{i}^{\infty}$ & 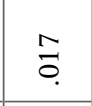 & f & $\stackrel{\Perp}{\circ}$ & $\exists$ & $\stackrel{\infty}{\circ}$ & 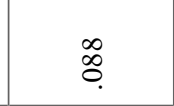 & 品 & $\stackrel{a}{i}$ & $\stackrel{\infty}{\rightarrow}$ \\
\hline$\stackrel{\infty}{\circ}$ & 苞 & $\begin{array}{l}+ \\
\infty \\
\infty\end{array}$ & $\stackrel{\infty}{\sim}$ & $\stackrel{?}{\rightarrow}$ & $\stackrel{\circ}{\circ}$ & డొ & : & 望 & $\bar{~}$ & $\cong$ & $\tilde{\sigma}$ \\
\hline 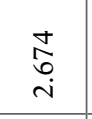 & în. & $\underset{i}{\text { Y }}$ & 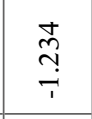 & 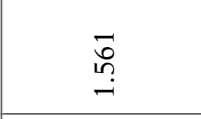 & $\overrightarrow{\vec{r}}$ & $\stackrel{\widetilde{f}}{i}$ & $\stackrel{m}{f}$ & $\stackrel{m}{m}$ & $\underset{d}{o}$ & 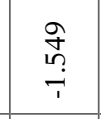 & $\stackrel{\circ}{\underset{N}{N}}$ \\
\hline$\stackrel{\infty}{\sim}$ & ơ & $\stackrel{\sim}{a}$ & $\stackrel{\circ}{\stackrel{9}{*}}$ & $\stackrel{n}{\exists}$ & ț & 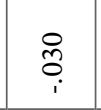 & $\tilde{\tilde{o}}$ & ฮั. & $\stackrel{t}{\mathrm{~g}}$ & $\stackrel{\circ}{\ni}$ & $\stackrel{\overbrace{}}{\dddot{\tau}}$ \\
\hline 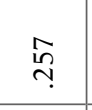 & $\overrightarrow{\mathrm{o}}$ & $\stackrel{\infty}{\circ}$ & $\stackrel{\overbrace{}}{\stackrel{\partial}{i}}$ & $\stackrel{\Xi}{\rightleftarrows}$ & ta & $\hat{o}_{i}^{r}$ & 寺 & ठे. & $\stackrel{t}{\mathrm{O}}$ & $\stackrel{\Im}{i}$ & $\underset{f}{f}$ \\
\hline 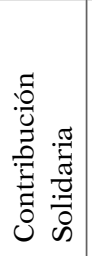 & 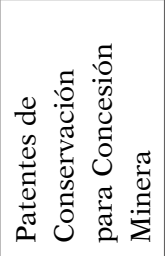 & 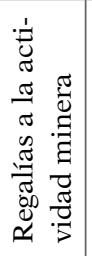 & 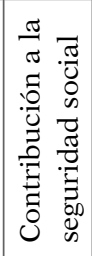 & 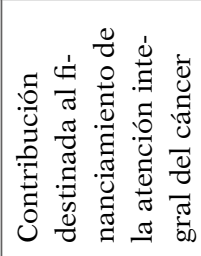 & 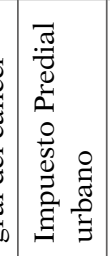 & 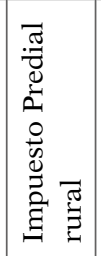 & 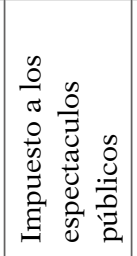 & 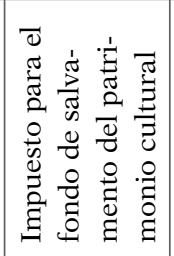 & 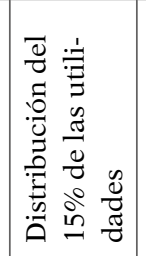 & 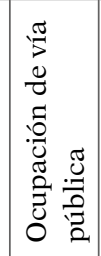 & 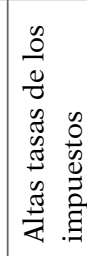 \\
\hline
\end{tabular}




\subsection{Efectos de la carga y presión tributaria por las restricciones de la pandemia Covid-19}

Los datos muestran que los ingresos de los contribuyentes en el 2020 fueron medios del $62.9 \%$, bajos del $26.7 \%$ y alto del $10.4 \%$. La aplicación de regresión lineal muestra que los niveles medios de ingresos influyen (Sig. $=0.006$ ) en el monto de los impuestos a pagar en un $2 \%(\mathrm{R} 2=0.024)$, también, los cambios que pueden sufrir los ingresos por la pandemia de Covid-19 incrementaría significativamente (sig. 0.006) el pago de impuestos en un $15.6 \%$. Por tanto, H1d la presencia de un factor externo como el Covid-19 influye significativamente en los ingresos (gráfico 3).

\section{Gráfico 3. Efecto de carga y presión tributaria}

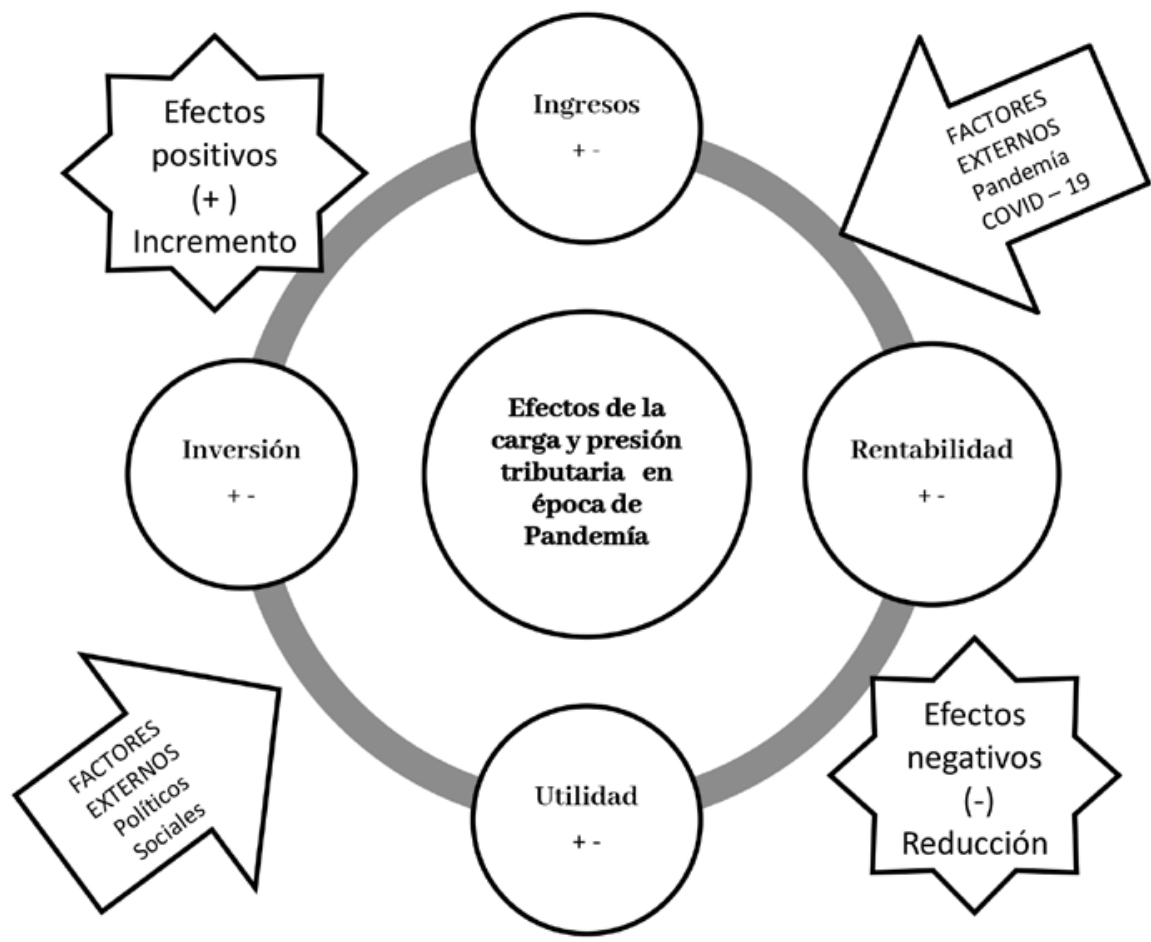

Fuente: Elaboración propia.

\section{Discusión y conclusiones}

Los resultados permiten corroborar que "existe evidencia empírica que demuestra el efecto negativo que tiene los impuestos en la economía" Serrano $(2009$, p.133) y existen "efectos negativos significativos de los impuestos en la actividad económica" Blanchard y Perotti (2002, p. 1329); por otro lado, las variaciones en las tasas de los impuestos puede tener "influencia positiva en la inversión extranjera directa" (Klemm \& Van, 2010, p. 5), sin embargo no ocurre lo mismo con las inversiones internas.

En ese contexto, se evidencia que la carga y presión tributaria tiene un efecto negativo en los ingresos, rentabilidad e inversión; en cambio, si estos fueran reducidos, contribuirían a la mejora no solo de los ingresos y ganancias sino que principalmente se podría convertir en un elemento motivador para la inversión, como mencionan Cardoso y Funchal (2011, p. 152) el "efecto de la regulación tributaria sobre las inversiones, los resultados demuestran que hay significación estadística y que una reducción 
de la carga tributaria, medida por la tributación sobre las ganancias empresariales, puede elevar los niveles de inversión".

Por otro lado, la presencia de efectos significativos sobre la inversión de los contribuyentes, evidencia que "la presión tributaria tiene efectos significativos en la inversión privada” Brito-Gaona e Iglesias (2017, p.153) y de Caballero y López (2012) que concluyeron que el "impuesto sobre la Renta y el Impuesto al Valor Agregado parecen tener una relación directa y no inversa con la inversión privada" (p. 54); sin embargo, es cierto que los "Impuestos a la Renta tienen un efecto perverso sobre las decisiones de inversión privada,por tanto, sobre el crecimiento económico y el empleo" (Caballero \& López, 2012, p. 62) como también se encontró en la presente investigación.

Finalmente, existe en $\mathrm{H} 2$ una relación directa entre recaudación tributaria y ciclo económico, porque tanto la presión como la carga tributaria recae en la recaudación. Existe una relación directa entre la recaudación tributaria y el PIB en un $63.2 \%$ y explica el $39.9 \%\left(\mathrm{R}=0.632, \mathrm{R}^{2}=0.399\right)$ existiendo una dependencia (Sig. 0.000); es decir, si existe un incremento en el PIB también existe un incremento en la recaudación o viceversa, aunque puede ser proclivo, como también ocurren en otros países (gráfico 4). Sin embargo, existen problemas para la recaudación debido a factores, como "la estructura económica y el nivel de desarrollo, las instituciones políticas, los aspectos culturales e ideológicos y la relación entre Estado y sociedad" (Gómez, 2009, p. 36) la intervención del Estado es importante para generar políticas tributarias que regulen la carga y presión tributaria que mejore la economía, a través de la creación de una "cultura tributaria basada en el control de la gestión, integración de la información y la equidad social” (Mejía et al., 2019, p. 1152).

\section{Gráfico 4. Ciclo económico PIB y recaudación tributaria (2000-2020)}

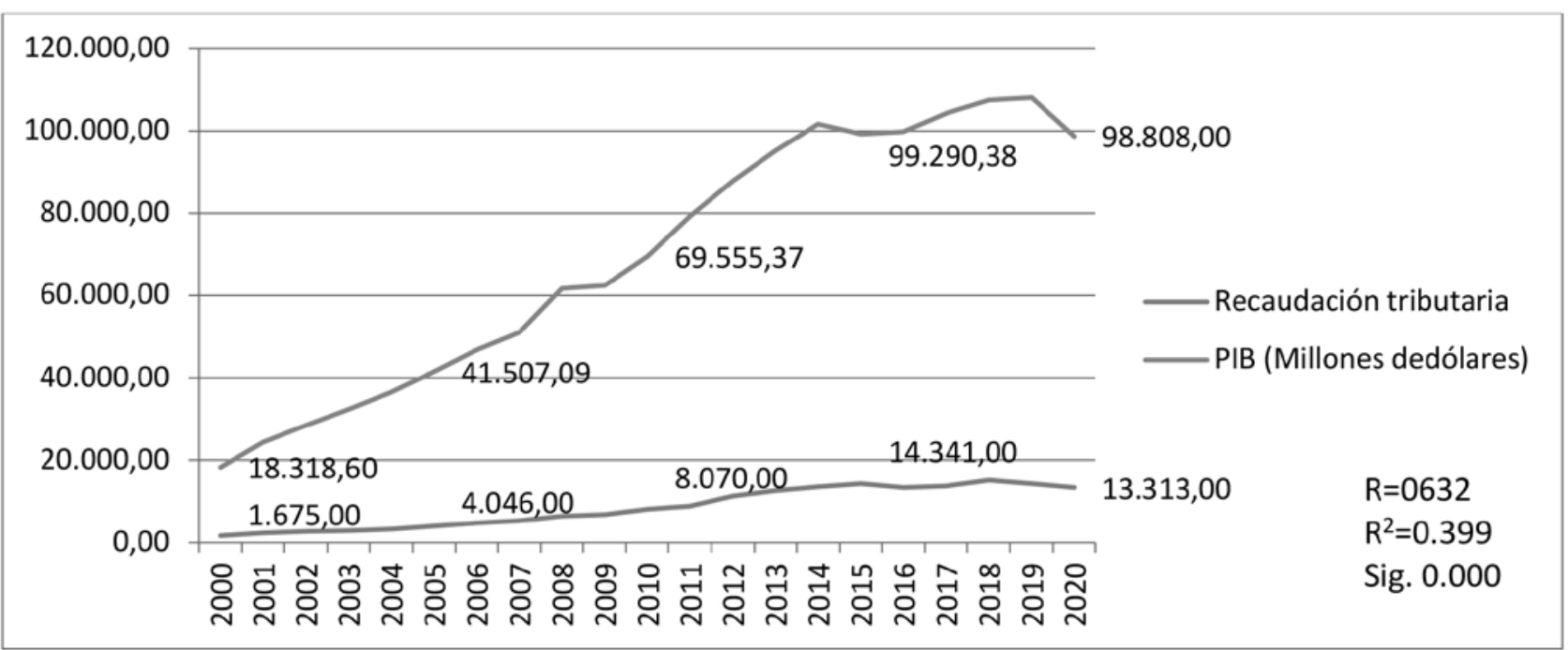

Fuente: Elaboración propia. INEC, 2021.

Por tanto, se demuestra que la carga y presión tributaria tiene un impacto negativo en la liquidez, rentabilidad e inversión es cierta; porque una variación en las tasas y en el número de impuestos influye significativamente en el incremento o reducción, dado que una alta carga impositiva no solamente reduce la liquidez y rentabilidad sino también la inversión, afectando al ciclo económico. Lo que significa que el impacto de la carga tributaria no solo está relacionado con el impacto sobre la distribución de la renta real de los impuestos (Pablo et al., 2006) sino también con el impacto sobre el sujeto (contribuyente fiscal) que "soporta realmente la carga del impuesto provocando 
que su rentabilidad se vea disminuida por el efecto impositivo" (Sarmiento, 2010, p. 208) ya que presupone una reducción por la disponibilidad de efectivo para el pago de los tributos. De este modo la investigación demuestra que la recaudación y el desempeño empresarial depende de las variaciones en la carga y presión tributaria que tienen los contribuyentes, y tiene una relación directa con el ciclo económico, porque el hecho del incremento en las obligaciones tributarias puede afectar en las utilidades e inversión de forma positiva o negativa como en la economía, ya que uno de los factores de crecimiento económico está relacionado con desarrollo empresarial y esto solo puede ocurrir si existe un éxito empresarial que dependerá de factores como la:

Cultura organizacional, la forma en la que reaccionaron a las condiciones del entorno, su disponibilidad de recursos, las decisiones que toman, la reducción del riesgo y el aprovechamiento de las oportunidades de negocio que tienen. (Tapia-Alba \& Chiatchooua, 2021, p. 9)

En la investigación se demuestra que la presión y carga tributaria tiene un efecto negativo, sin embargo los incentivos fiscales pueden provocar un efecto positivo:

Ya que al no pagar impuestos la liquidez se incrementa, contando con ello con más recursos para hacer frente a compromisos. Por otra parte, la solvencia se mejore, ya que disminuye en una cantidad importante los pasivos por Impuesto a la Renta. (Yaguache et al., 2019, p. 377)

Esto repercute en los ingresos de la economía; por lo que el ciclo económico depende también de las recaudaciones, que tiene un efecto positivo y negativo significativo en la actividad y desempeño empresarial de los contribuyentes y no solo en la recaudación tributaria, sino que al mismo tiempo repercute en el ciclo económico de un país, puesto que se determina que existe una relación directa.

Por lo tanto, se concluye:

El tipo y número de impuestos que está obligado a pagar como contribuyente del RISE, RG o especial tiene un efecto en la disminución de la inversión, utilidad e ingresos.

Las variaciones del Impuesto a la Renta, Contribución solidaria y el aumento de las tasas de los impuestos disminuye a inversión significativamente, en cambio el Impuesto al Valor Agregado estimula las inversiones.

Se produciría una reducción en utilidades por cambios en el Impuesto a la Salida de Divisas, Contribuciones Solidarias, Contribución a la financiación del cáncer, y un incremento en las tasas de los impuestos; sin embargo, el Impuesto a las Patentes incrementaría las utilidades.

Existe un efecto negativo en los ingresos de los contribuyentes como consecuencia de los cambios en el Impuesto para el Fondo de Salvamento del Patrimonio Cultural, ocupación de vía pública, altas tasas de los impuestos; en cambio es positivo, cuando exista un aumento en las Patentes de Conservación para Concesión Minera, Impuesto de patentes municipales, Impuesto para el fondo de salvamento del patrimonio cultural, Ocupación de vía pública.

Se evidencia que la presión tributaria tiene un efecto significativo en la inversión de los contribuyentes y existe una relación directa entre la recaudación tributaria y el ciclo económico en el Ecuador.

Las limitaciones de esta investigación están relacionadas con el acceso a datos actualizados y desagregados, porque el limitado acceso a información contable de los contribuyentes disponible principalmente de quienes no están obligados a llevar contabilidad, ha hecho que este segmento no sea considerado en la investigación, lo que representa la necesidad de profundizar y ampliar el estudio a este segmento y a otras regiones del Ecuador a fin de comparar los resultados y llegar a una conclusión generalizada. 
Una de las posibles líneas de investigación creadas por este estudio es el abordaje y profundización del comportamiento del contribuyente en el contexto de la tributación de impuestos a nivel nacional.

\section{Referencias}

Alcívar, A., \& Saines, A. (2011). Análisis de la quiebra empresarial de Pequeñas y Medianas Empresas en Ecuador (2006-2010). Una aplicación del Modelo de Duración de Cox (1972). https://bit.ly/3hbm4GR

Archel, P., \& Goméz, M. (2014). Crisis de la valoración contable en el capitalismo cognitivo. Revista Innovar Journal, 103-116. https://bit.ly/3AlnHsX

Asamblea Nacional del Ecuador (2018). Ley de Régimen Tributario Interno, LRTI. Registro Oficial Suplemento $\mathrm{N}^{\mathrm{a}} 463$ del 17 de noviembre de 2004. https://bit.ly/3dAHqLq

Belloso, R. (2010). Efectos de la aplicación del Impuesto a las Transacciones Financieros en los principios de no confiscatoriedad y capacidad contributiva. Revista Electrónica Arbitrada Comercium et Tributum, II (1). https://bit.ly/3AnGRye

Blanchard, O., \& Perotti, R. (2002). And empirical characterization of the dinamic effects og changes in government spending an taxes on output. The Quarterly Journal of Economic, 117(4), 13291368. https://bit.ly/3An8aZR

Brito-Gaona, L., \& Iglesias, E. (2017). Inversión privada, gasto público y presión tributaria en América Latina. Revista Estudios de Economía, 44(2), 131-156. http://dx.doi.org/10.4067/S071852862017000200131.

Caballero, E., \& López, J. (2012). Gasto público, Impuesto sobre la renta e inversión privada en México. Revista Investigación Económica, 71(280), 55-84. https://bit.ly/2Trdri

Campo-Arias, A., \& Oviedo, H. C. (2008). Propiedades psicométricas de una escala: la consistencia interna. Revista de Salud Pública, 10(5), 831-839. https://bit.ly/2Trdri

Cardoso, R., \& Funchal, B. (2011). O efeito da regulacao trabalhis e tributaria nos investimentos no Brasil. Revista Adm, MACKENSIEm 12(4), 127-154. https://bit.ly/3wcd1cT

Chávez, L., \& López, V. (2019). Determinantes del recaudo del impuesto a la propiedad inmobiliaria: un enfoque estadístico para México. Revista Iberoamericana de estudios municipales, 19, 89-119. http://dx.doi.org/10.4067/S0719-17902019000100089 .

Chen, S., Chen, X., Cheng, Q., \& Shevlin, T. (2010). Are Family firm more tax aggressive than non-family firms? Revista Journal of Financial Economic, 41-61. https://doi.org/10.1016/j.jfineco.2009.02.003.

Congreso Nacional (Modificado 21 de agosto de 2018). Código Tributario. https://bit.ly/3jAwsJN

Crespo, A. (2016). La presión fiscal en el Ecuador: Análisis de la carga impositiva real. (Tesis de Maestría). Guayaquil.

Díaz-LLanes, M. (abril-junio 2012). Inmovilizado: El punto de partida de la liquidez. Revista Universo Contábil, 8(2), 134-145. https://doi.org/10.4270/ruc.2012217

Fernández, E. (2004). Los factores condicionantes de la presión fiscal empresarial española a partir de la información contable. Especial mención a las decisiones financieras. Revista Española de Financiación y Contabilidad, 3, 125-159. https://doi.org/10.1080/02102412.2004.10779516

Gómez, J. (2009). La economía política de la política tributaria en América Latina. Chile: 21 Seminario regional de política fiscal: ILPES/CEPAL.

INEC (2017). Panorama Laboral y Empresarial del Ecuador. Quito : Laboratorio de Dinámica Laboral y Empresarial. https://bit.ly/3j0iZtZ

Hall, R., \& Dale, J. (1967). Tax policy and investment behavior. Revista The Amrican Economic Review, 391-414. https://bit.ly/2SJgT7v

Klemm, A., \& Van, Stefan (2009). Empirical Evidence on the Effects of Tax Incentives

IMF Working Paper, WP/09/136. Internacional Monetary Found, 1-26.

Landázuri, O. (28 de 07 de 2019). En Ecuador, la carga tributaria llega al 20,4\% del PIB, indica un estudio. El Universo. https://bit.ly/3xpORNH 
Lima, E., \& Resende, A. (2019). Um estudo sobre a evolucao da carga tributaria no Brasil: uma análise a partir da Curba de Laffer. Revista INTERACOES, Campo Grande, MS, 20(1), 239-255. http://dx.doi.org/ 10.20435/inter.v0i0.1609 .

Mejía, O., Pino, R., \& Parrales, C. (2019). Políticas tributarias y la evasión fiscal en la República del Ecuador. Aproximación a un modelo teórico. Revista Venezolana de Gerencia (RVG), 24(88), 1147-1165. https://doi.org/10.37960/revista.v24i88.30169.

Monterrey, J., \& Sánchez, A. (2015). Planificación fiscal y gobierno corporativo en las empresas cotizadas Españolas. Revista Hacienda Pública Española. Review of Public Economics, 214, 55-90. https:// bit.ly/3jEx $8 x B$

Monterrey, J., \& Sánchez, A. (2017). Los impuestos como determinantes de la inversión empresarial. Evidencia empírica en empresas españolas que no cotizan en bolsa. Revista de Contabilidad, 20(2), 195-209. https://bit.ly/3qGvPjf

Monterrey, J., \& Sánchez, A. (2020). ¿Cómo ha evolucionado la presión fiscal de la empresa española en la última década? Remedios normativos frente a la planificación fiscal. Revista de Contabilidad - Spanish Accounting Review, 23(2), 224-237. https://doi.org/10.6018/rcsar.370841

Nava, M. (2009). Análisis financiero: una herramienta clave para una gestión financiera eficiente. Revista Venezolana de Gerencia, 14(48), 606-628. https://bit.ly/3ygMBs4

OCDE, CIAT, IDB, ECLAC, DEV (2020). Estadísticas tributarias en América Latina y el Caribe 2020. 1990-2018. Paris. https://bit.ly/367f2fV

Pablo, M., Cansino, J., Castro, M., Román, R., \& Yñiquez, R. (2006). 100 ejercicios de economía pública. Delta.

Parada, J. (1988). Rentabilidad empresarial. Un enfoque de gestión. Universidad de Concepción. https://bit. ly/2UleCzH

Pecho, M., \& Peragón, L. (2013). Estimación de la carga tributaria sobre la inversión en América Latina. https:// bit.ly/365ad6K

Piedra, A., Salinas, J., \& Vázquez, J. (2016). Caracterización tributaria en barrios urbanos populares: caso Parroquia el Vecino. Revista Retos, 11(1), 88-104. https://doi.org/10.17163/ret. n11.2016.06.

Quispe, G., Arellano, O., Rodríguez, E., Negrete, A., \& Vélez, K. (2019). Las reformas tributarias en el Ecuador. Análisis del periodo 1492 a 2015. Revista Espacios, 4(13), 21. https://bit.ly/3qHAyl1

Quispe, G., Arellano, O., Rodríguez, E., Vélez, K., \& Negrete, O. (2017). La carga tributaria y su efecto en los contribuyentes de la ciudad de Riobamba (periodo 2015-2016). En CIDE, Cátedra Iberoamericana de Contabilidad y Auditoria, 26-39. https://bit.ly/3hybrgm

Roca, S., Simabuko, L., \& Dyer, J. (2004). Impacto tributario en el servicio de transporte terrestre interprovincial de pasajeros: el caso del retiro de la exoneración del IGV. Revista Cuadernos de Difusión, 916), 21-52. https://bit.ly/3hp4sWR

Rodríguez, J., \& Ávila, J. (2017). La carga tributaria sobre los ingresos laborales y de capital en Colombia: El caso del Impuesto sobre la Renta y el IVA. Revista Cuadernos de Economía, 36(72), 99-138. https://doi.org/10.15446/cuad.econ.v36n72.65877.

Ruiz-Vargas, M., \& Navarro-Morato, O. (2016). Incidencia de la política de incentivos tributarios sobre la inversión en el sector minero energético Colombiano: un análisis exploratorio de su efectividad. Revista Cuadernos Contabilidad, 17(43), 109-126. https://doi.org/10.11144/Javeriana. cc17-43.ipit.

Salto, I., Tenesaca, M., Arroba, J., \& Villalta, E. (2018). Los beneficios tributarios su incidencia en la liquidez y la rentabilidad de las Asociaciones de Economía Popular y Solidaria. Revista Ciencia, Técnica y Mainstreaming social, 1, 115-124. https://doi.org/10.4995/citecma.2018.9855.

Sarmiento, J. (2010). Identificación del impacto de la carga fiscal en ls pymes de Bogotá, a partir del contexto Latinoamericano, nacional y regional. Cuadernos de Contabilidad, 11(28), 201-237. https://bit.ly/3xeuF0S

Serrano, J. (2009). Análisis dinámico de la recaudación tributaria en el Ecuador aplicando modelos VAR. Revista Fiscalidad , 123-167. 
SRI (2020). Servicio de Rentas Internas (SRI). https://bit.ly/3xQAotr

Superintendencia de Compañías (2020). Superintendencia de Compañías del Ecuador. https://bit. ly/2UkG4Oa

Tapia-Alba, J., \& Chiatchooua, C. (2021). Análisis de la relación entre actividad empresarial y desigualdad económica. Revista CEA, 7(14) e1595, 1-25. https://doi.org/10.22430/24223182.1597.

Yaguache, M., Higuerey, A., \& Inga, E. (2019). Incentivos fiscales, liquidez y solvencia en las empresas del Ecuador. Revista Venezolana de Gerencia, 24 (número especial 2), 631-378. https://doi. org/10.37960/revista.v24i2.31498.

Zamora, A. (2008). Rentabilidad ventaja comparativa: un análisis de los sistemas de producción de Guayaba en el Estado de Michoacan. https://bit.ly/3wfmqAz 